Radial and Nonradial Pulsations as Probes of Stellar Physics

ASP Conference Series, Vol. 259, 2002

C. Aerts, T.R. Bedding, \& J. Christensen-Dalsgaard, eds.

\title{
Interrelation of Modal Amplitudes of Double-Mode Pulsators
}

\author{
L. Szabados \\ Konkoly Observatory, Budapest, Hungary
}

\begin{abstract}
During the study of the factors governing the modal amplitudes of the double-mode radial pulsators, it has been found that the $R_{21}$ Fourier parameter deduced from the light curves is in a relationship with the ratio of the modal amplitudes, for beat Cepheids and RR Lyrae variables, as well. The form of this relation gives a natural explanation for the fact that double-mode pulsators with slightly excited second modes do not exist.
\end{abstract}

Beat pulsators, i.e., those stars in which two radial modes are excited simultaneously, are of special importance because the existence of two radial modes is a strong constraint for a viable theoretical model. Kolláth \& Buchler (2001) give a review on the recent progress in modelling double-mode stellar pulsation.

The amplitude of the brightness variation of the individual modes (i.e., the mode energy content) has been a neglected phenomenological property of the double-mode pulsating variables although it means a further constraint on the realistic models describing the pulsations in these stars.

The aim of this paper is to find the relationship between the modal amplitudes to get an idea about the controlling factors of the mode energy. The study is based on 15 beat Cepheids in the Milky Way galaxy (among the known 17 such double-mode pulsators) and 93 newly detected double-mode Cepheids in the SMC. The SMC sample of double-mode Cepheids is based on the extensive OGLE data-set (Udalski et al., 1999a,b). Recently the OGLE-data on the beat Cepheids in the LMC also became available (Soszyński et al., 2000). This latter sample contains 76 double-mode Cepheids.

While the observational data are homogeneous for the Magellanic Cloud Cepheids, in order to reach the most homogeneous data set for the Galactic double-mode Cepheids, data obtained by various observers in the Johnson and/or Kron-Cousins $U B V R$ system have been taken into account.

The observational data on double-mode RR Lyrae stars analysed in this paper comprise the publicly available MACHO LMC-sample (75 stars, Alcock et al., 1997) and an earlier and smaller sample of RRd stars in the globular cluster IC 4499 (13 stars, Clement et al., 1986).

Correlations between various phenomenological parameters were searched for. The parameters investigated have been those determined by the standard method of Fourier-decomposition. Finally, an interesting relation between the modal amplitudes of beat Cepheids and RR Lyrae stars has been found. There is a tendency to have a smaller amplitude for the higher excited overtone with increasing $R_{21}$ Fourier parameter of either excited mode. This feature is common for both Galactic and Magellanic Cloud samples. Since the harmonics of the 

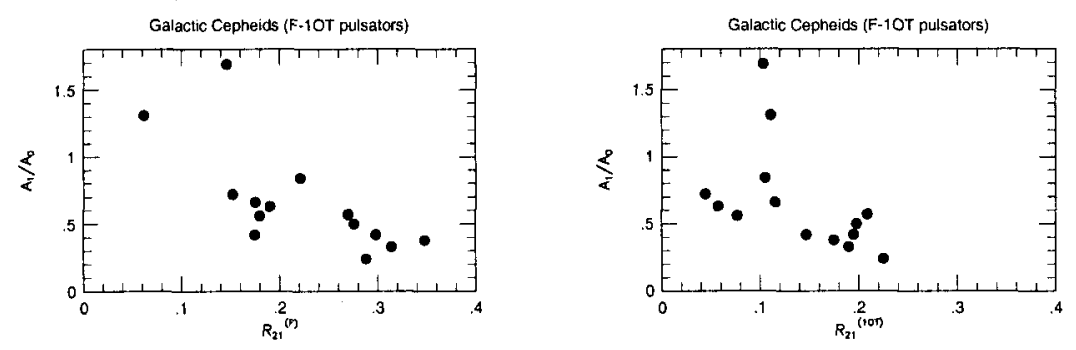

Figure 1. The ratio of the modal amplitudes for Galactic Cepheids. The abscissa of the left panel is the $R_{21}$ Fourier parameter for the fundamental mode oscillation, while that of the right panel is $R_{21}$ of the first overtone.

individual modes have lower amplitudes with respect to the main oscillations, i.e., the value of $R_{21}<1$, the relationship between the mode amplitude ratios and the $R_{21}$ Fourier parameters offers an obvious explanation why a "slightly" excited second mode does not exist among the double-mode radial pulsators. If a second mode is excited, its amplitude is not infinitesimal.

Fig. 1 shows the dependence of the mode amplitude ratio on $R_{21}$ for the Galactic beat Cepheids. A similar tendency exists for the double mode Cepheids and RR Lyrae stars in the Magellanic Clouds and in the globular cluster studied. This suspected relation between the $R_{21}$ Fourier parameter and the mode energy content deserves detailed theoretical studies for both supporting existence of such a relationship and to reveal the underlying physical mechanism. The calculations performed by Kolláth \& Buchler (2001, their Fig. 9) are in accord with the empirical relationship of the present study.

Acknowledgments. The financial support from the OTKA T029013 and T034584 grants as well as the IAU travel support are gratefully acknowledged.

\section{References}

Alcock, C., Allsman, R.A., Alves, D., et al. (The MACHO Collaboration) 1997, ApJ, 482, 89

Clement, C.M., Nemec, J.M., Robert, N., Wells, T., Dickens R.J., \& Bingham, E.A. 1986, AJ, 92,825

Kolláth, Z. \& Buchler, J. R. 2001, in Stellar Pulsation - Nonlinear Studies, eds. M. Takeuti \& D. Sasselov, ApSS Library, Vol. 257, 29

Soszyński, I., Udalski, A., Szymański, M., et al. 2000, Acta Astron., 50, 451

Udalski, A., Soszyński, I., Szymański, M., et al. 1999a, Acta Astron., 49, 1

Udalski, A., Soszyński, I., Szymański, M., et al. 1999b, Acta Astron., 49, 437 